\title{
Refined toric branes, surface operators and factorization of generalized Macdonald polynomials
}

\author{
Yegor Zenkevich \\ Institute for Theoretical and Experimental Physics (ITEP), \\ Moscow 117218, Russia \\ National Research Nuclear University MEPhI, \\ Moscow, 115409, Russia \\ Physics Department, Moscow State University, \\ Moscow 117312, Russia \\ Dipartimento di Fisica, Università di Milano-Bicocca, \\ Piazza della Scienza 3, I-20126 Milano, Italy \\ INFN, sezione di Milano-Bicocca, \\ I-20126 Milano, Italy \\ E-mail: yegor.zenkevich@gmail.com
}

AbStRaCT: We find new universal factorization identities for generalized Macdonald polynomials on the topological locus. We prove the identities (which include all previously known forumlas of this kind) using factorization identities for matrix model averages, which are themselves consequences of Ding-Iohara-Miki constraints. Factorized expressions for generalized Macdonald polynomials are identified with refined topological string amplitudes containing a toric brane on an intermediate preferred leg, surface operators in gauge theory and certain degenerate CFT vertex operators.

Keywords: Topological Strings, Conformal and W Symmetry, M(atrix) Theories

ARXIV EPRINT: 1612.09570 


\section{Contents}

1 Introduction $\quad 1$

1.1 Refined topological strings and branes 3

1.2 q-deformed CFT 5

2 Factorization of generalized Macdonald polynomials $\quad 6$

2.1 Schur and Macdonald polynomial factorization. A reminder 6

2.2 Generalized Macdonald polynomials factorization on the general topological locus

2.3 Factorization identities from matrix model averages

2.4 New formulas for skew generalized Macdonald polynomials 9

$\begin{array}{ll}2.5 & \text { Gluing, traces and factorization of instanton sums }\end{array}$

3 Toric brane on the intermediate leg and surface operators 12

$\begin{array}{ll}3.1 & \text { Refined topological amplitudes with branes } \\ & \text { Degen }\end{array}$

$\begin{array}{lll}3.2 & \text { Degenerate fields and surface operators } & 15\end{array}$

4 Conclusions and further prospects $\quad 16$

\section{Introduction}

The interplay between algebraic structures and geometry has been fundamental to the development of mathematics in the recent decades. In particular, it has led to a cornucopia of new results in mathematical physics. One of examples is the (refined) topological vertex function [1-9] which on the geometric side describes Gromov-Witten and DonaldsonThomas invariants of toric Calabi-Yau (CY) three-folds, while from the algebraic point of view it is the intertwiner of the Ding-Iohara-Miki (DIM) algebra ${ }^{1}[10,11]$. The second famous example comes from the gauge theory: the equivariant cohomology of the instanton moduli spaces (captured by Nakajima quiver varieties [12-14] and the corresponding Nekrasov partition functions [15-22]) is acted on by a certain vertex operator algebra, which turns out to be the $W_{N}$-algebra of two dimensional conformal field theory. This correspondence between the geometric (moduli space) and algebraic ( $W_{N}$-algebra) objects is known as the AGT relation [23-25] and has many known implications and generalizations [26-36]. These two examples are in fact directly related to each other and their relation can be understood on both sides of the algebro-geometric correspondence. On the algebraic side the equivariant cohomology (or more precisely $K$-theory [37]) of the instanton moduli spaces is a tensor product of Fock representations of the DIM algebra [38], while the $q$-deformed

\footnotetext{
${ }^{1}$ Alternative names include quantum toroidal, elliptic Hall, spherical degenerate DAHA algebras, or simply $U_{q, t}\left(\widehat{\widehat{\mathfrak{g l}}}_{1}\right)$.
} 
$W_{N}$-algebra generators are built from the currents of the DIM algebra [39-41] and vertex operators are combinations of topological vertices intertwining the action of the DIM algebra and therefore of the $W_{N}$-algebra [42]. On the geometric side the $5 d$ gauge theory is obtained by compactifying M-theory on the toric CY three-fold. The parameters of the gauge theory correspond to Kähler moduli of the CY and the cohomology of the moduli space of instantons is identified with the Hilbert space of M2 branes stretching between toric fixed points.

In this paper we explore a particular case of the algebro-geometric correspondence, which is important for topological strings as well as for gauge theories. We consider refined toric branes wrapping Lagnangian submanifolds inside a toric CY three-fold [43]. As is well-known, this setup corresponds to surface operators in gauge theory and to degenerate fields of the $W_{N}$-algebra [44-50,53-55]. We will consider mostly the algebraic side of the problem and relate the stack of refined branes on the preferred leg of the toric diagram to a particular intertwining operator of DIM algebra, which can be recast into a combination of generalized Macdonald polynomials $[56,59]$. The properties of the branes are related to the remarkable factorization identities for generalized polynomials evaluated on a particular submanifold in the brane moduli space called the topological locus.

Generalized Macdonald polynomials [56, 59] play the central role in the AGT correspondence. They arise naturally in the study of the DIM algebra representations on tensor producs of Fock modules. In [59, 60] matrix elements between generalized Macdonald polynomials were computed using matrix model techniques. They turned out to reduce to integral factorization identities, which provide a very explicit answer for the $q$-Selberg averages in terms of Nekrasov functions.

In this note we would like to use these integral identities to prove new topological locus factorization identity recently found in [61]. For special values of parameters the integrals disappear and one is left with Macdonald polynomials evaluated at the topological locus. The integral identity implies that those are still given by the factorized formulas. This technique allows us to find several new identities for generalized Macdonald polynomials on a more general topological locus. In this way we prove and generalize the results of [61].

We then connect the factorization of the polynomials to the refined topological string picture. To this end we interpret matrix model averages as topological string amplitudes on toric CY threefolds with Lagrangian branes appropriately placed on the legs of the toric diagram (for exact correspondence and explanation see [60]). Factorization of averages relies on the particular properties of the branes residing on the preferred direction of the diagram. The topological locus corresponds to a certain degenerate limit of the CY, which models addition of a stack toric branes on one of the legs in the preferred direction. Factorization of generalized Macdonald polynomials in this picture allows us to understand the amplitudes with toric branes placed on intermediate preferred legs of the toric diagram.

One can also interpret factorization formulas for generalized Macdonald polynomials in terms of CFT vertex operators in Dotsenko-Fateev (DF) representation. In this case the radical simplification of the formulas occurs due to the particular choice of the dimensions for which the vertex operators do not require any screening currents. In view of the AGT relations this corresponds to a particularly simple surface operator in the corresponding gauge theory. 
In the remaining part of the introduction we discuss the main points of this network of correspondences in more detail. In section 2 we write down and prove the factorization identities, in section 3 we connect this results with topological strings and gauge theories. We present our conclusions in section 4 .

\subsection{Refined topological strings and branes}

Refined topological string theory $[8,9]$ is a deformation of the topological string theory living on a toric CY three-fold, which gives additional information on the spin content of the D-brane BPS spectrum of type IIA string theory. Refined amplitudes are computed using refined topological vertex, quite similarly to the ordinary topological vertex computations [1-7]. A Young diagram $Y_{i}$ is assigned to each leg $i$. The vertices, always trivalent, correspond to certain explicit combinations $C_{Y_{i} Y_{j} Y_{k}}(q, t)$ of symmetric functions depending on three Young diagrams $Y_{i, j, k}$ on the adjacent legs $i, j, k$ :

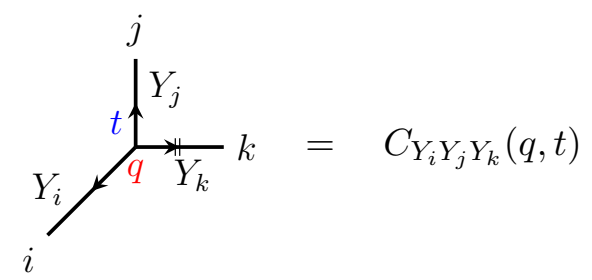

There is one crucial point in the computation of refined amplitudes. Unrefined topological vertex $C_{Y_{i} Y_{j} Y_{k}}(q, q)$ is cyclically symmetric in the three Young diagrams $Y_{i}, Y_{j}, Y_{k}$, while the refined vertex $C_{Y_{i} Y_{j} Y_{k}}(q, t)$ for general $q \neq t$ is not. The recipe above, therefore, includes the choice of ordering of Young diagrams in each vertex. This choice is indicated by the double ticks and the labels $q$ and $t$ on the corresponding legs in eq. (1.1). In what follows we will usually omit the indices $q$ and $t$. It turns out that the choices for the neighbouring vertices should be coordinated, so that the only freedom remaining is the global choice of the preferred direction (horizontal in eq. (1.1)) on the toric diagram. We omit here the concrete expression for $C_{Y_{i} Y_{j} Y_{k}}(q, t)$, which can be easily found in the literature, not to overcomplicate our presentation.

To get the final answer for the amplitude one takes the sum over all the Young diagrams $Y_{i}$ on the intermediate legs, each taken with weight ${ }^{2}\left(-Q_{i}\right)^{\left|Y_{i}\right|}$, where $Q_{i}$ denotes the exponentiated complexified Kähler parameter of the two-cycle associated to the leg $i$. Let us give the simplest example of two vertices glued together to form the resolved conifold geometry:

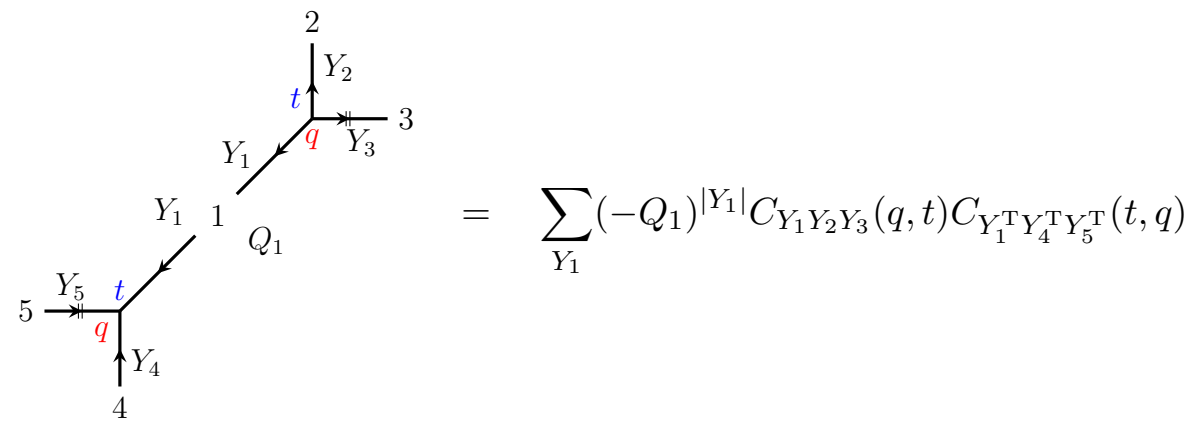

\footnotetext{
${ }^{2}$ In general there are also framing factors which we will not need here.
} 
With the external lines one can associate either empty or non-empty diagrams which do not take part in the sums. The former choice gives the closed string amplitude (partition function), while the latter one gives the open string amplitude with stacks of toric branes on the external legs determining the external diagrams, or "boundary conditions" for the theory:

$$
\begin{aligned}
\underbrace{}_{Q_{1}}= & =\sum_{Y_{1}}(-Q)^{\left|Y_{1}\right|} C_{Y_{1} \varnothing W}(q, t) C_{Y_{1} \varnothing \varnothing}(t, q) \\
& =Z_{\text {open }}\left(\varnothing_{\varnothing}^{\varnothing} W \mid Q_{1}\right) \\
Z_{\text {closed }}\left(Q_{1}\right) & =Z_{\text {open }}\left(\varnothing_{\varnothing}^{\varnothing} \mid Q_{1}\right)
\end{aligned}
$$

The dashed lines here denote toric branes. The number of branes in the stack sets the maximal possible number of rows in the Young diagram $W$. The final answer for the closed string amplitude does not depend on the choice of the preferred direction, though open string amplitudes do. ${ }^{3}$

In the unrefined case there is also a natural way to put a stack of toric branes on the internal leg (and indeed on any Lagrangian submanifold of the CY). However, in the refined case the study of branes on the external lines has been so far very limited (see [51], though). In the present paper we will address this problem and propose a recipe to put a stack of branes on the intermediate preferred leg. To do this we will employ the duality between open and closed string amplitudes.

Open-closed duality in topological strings allows one to model stacks of toric branes by closed string amplitudes [52-55]. The open string amplitudes should be packed in the Ooguri-Vafa generating function, and the closed strings propagate in the modified background containing additional vertical line in the toric diagram. Let us draw the dual pictures in the simplest case of one toric brane. The diagram corresponding to the brane can have at most one column, i.e. it is of the form ${ }^{4} W=[l]$. We then have

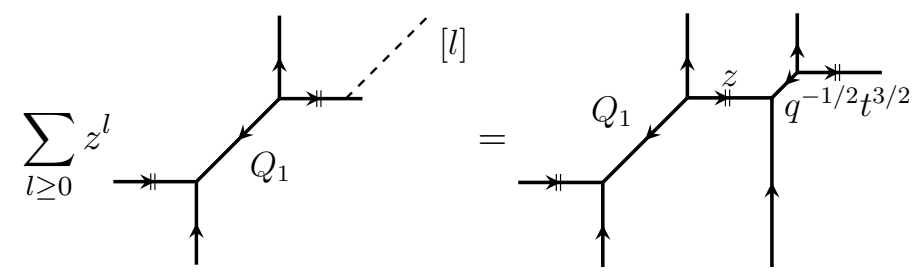

In the l.h.s. of eq. (1.5) $z$ plays the role of the holonomy of the (abelian) gauge field living on the toric brane, while on the r.h.s. it is identified with the Kähler parameter of the

\footnotetext{
${ }^{3}$ In the algebraic approach of [38] the choice of the preferred direction is associated with the choice of the slope of the coproduct $\Delta$ used in the definition of the DIM algebra. The most relevant choices used e.g. in $[62,63]$ where the "horizontal" coproduct $\Delta$ and the "vertical" (or perpendicular, or Drinfeld) coproduct $\Delta^{\perp}$.

${ }^{4}$ Compared to the notation of [55] we use the transposed diagram $W$. Another way to obtain our conventions from that of [55] is to exchange the equivariant parameters, $q \leftrightarrow t^{-1}$. Using the terminology of [43] this amounts to the exchange of $q$-branes and $\bar{t}$-branes.
} 
two-cycle obtained by adding an extra vertical line to the geometry. In general, for $N$ toric branes the Kähler parameter in the r.h.s. will change to $q^{-1 / 2} t^{N+1 / 2}$.

There are several points requiring clarification in this approach which are absent for ordinary topological string, i.e. for $t=q$, and appear only in the refined case:

1. The additional vertical line necessarily intersects all the parallel legs coming out of the diagram if there happen to be any (see figure $4 \mathrm{~b}$ ) for an example). One expects that the amplitude should be insensitive to these intersections since they have nothing to do with the toric brane insertion. However, in the refined case there is no way to make a "trivial crossing" of two lines: no choice of the Kähler parameter gives the desired result. One concludes that for several parallel legs the toric brane attached to one of them also interacts with all the others.

2. Although there is no way to make a "trivial crossing" of lines one can make a crossing, which models the trivial one in some situations. For example, this crossing can be used to set the diagram on one side to vanish if the diagram on the other vanishes (see figure $5 \mathrm{~b}$ ), c)). However, it works only in one direction: either the left diagram vanishes whenever the right one is empty or vice versa.

3. Because of these features of the refined theory it is unclear how to put a toric brane on the intermediate preferred leg.

The explanation of these puzzles will be the main focus of the present work. We will show that the amplitudes in the presence of the toric brane on the intermediate leg can be identified with generalized Macdonald polynomials evaluated on the topological locus.

\section{$1.2 q$-deformed CFT}

It was shown in [57-60], that certain refined topological string amplitudes on toric CY three-folds correspond to conformal blocks of the $q$-deformed Virasoro or $W_{N}$-algebras. ${ }^{5}$ The horizontal legs of the toric diagram represent the Hilbert space of the CFT, on which the conformal algebra acts, and the intersections with vertical legs give vertex operators or intertwiners of the algebra (see figure 1). Naturally, the sums over Young diagrams living on the horizontal lines represent the sums over the complete basis of states in the CFT Hilbert space. There is a natural choice for such a basis - the basis of generalized Macdonald polynomials, which leads to explicit factorized matrix elements for the vertex operators given by Nekrasov formulas. The sums over diagrams on the vertical lines corresponds to the integrals over the positions of the screening currents appearing in the Dotsenko-Fateev representation of the conformal blocks. Therefore, vertical lines correspond not simply to vertex operators, but more concretely to the screened vertex operator insertions $[41,60]$. The topological loci, i.e. the submanifold of the moduli space on which generalized Macdonald polynomials factorize into products of monomials, represent the special set of parameters, for which the number of screenings is zero.

\footnotetext{
${ }^{5}$ More concretely, to get a conformal block one should consider only balanced toric diagrams, see [40, 41] for details.
} 


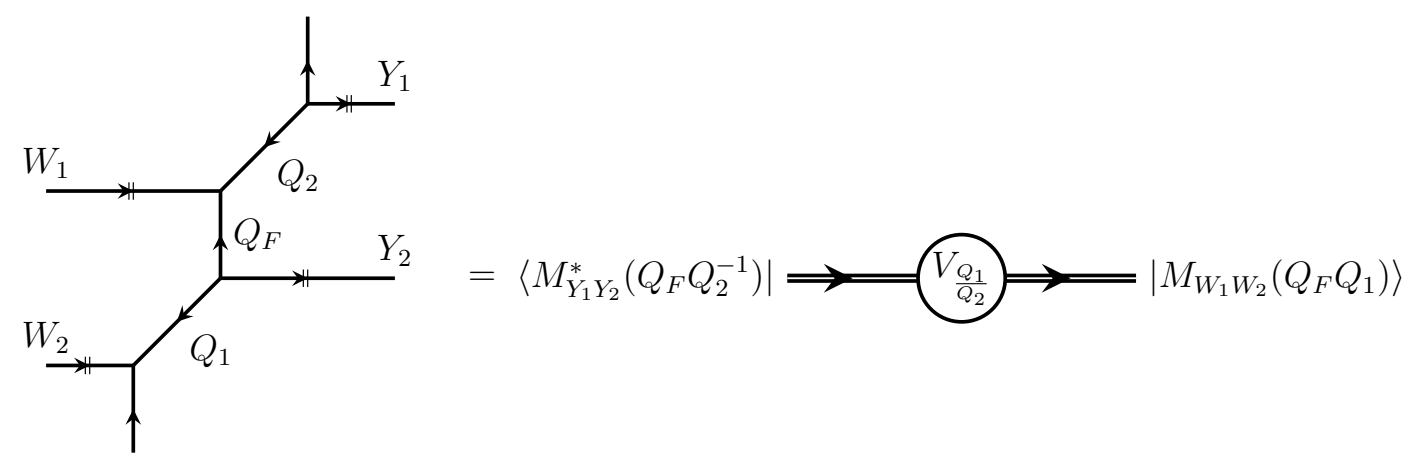

Figure 1. The correspondence between refined topological string amplitudes and vertex operators of CFT. Double lines in the r.h.s. denote the CFT Hilbert space on which $\operatorname{Vir}_{q, t} \oplus$ Heis algebra acts. On the l.h.s. it corresponds to the two horizontal lines. The circle in the r.h.s. represents the vertex operator corresponding to the intersection with the vertical line in the toric diagram in the 1.h.s. The matrix element on the r.h.s. is computed in the basis of generalized Macdonald polynomials $M_{Y_{1} Y_{2}}$, which corresponds to the choice of horizontal preferred direction (lines marked by by double ticks) on the l.h.s. Notice the relation between the Kähler parameters $Q_{1,2}, Q_{F}$ of the CY on the l.h.s. and the parameters of the vertex operator $V_{Q_{1} / Q_{2}}$ and the states on the r.h.s.

\section{Factorization of generalized Macdonald polynomials}

\subsection{Schur and Macdonald polynomial factorization. A reminder}

Let us first recall the familiar factorization formulas for Schur and Macdonald polynomials. Schur polynomials $s_{Y}\left(x_{i}\right)$ are symmetric polynomials in the variables $x_{i}, i=1 \ldots N$ labelled by Young diagrams $Y$. They can be understood as characters of finite-dimensional irreducible representations $\mathcal{R}_{Y}$ of $\mathfrak{s l}_{N}$ algebra corresponding to the Young diagrams $Y$ :

$$
s_{Y}(x)=\operatorname{tr}_{\mathcal{R}_{Y}} \operatorname{diag}\left(x_{1}, \ldots, x_{N}\right)
$$

We usually write all symmetric polynomials as functions of the power sums $p_{n}=\sum_{i=1}^{N} x_{i}^{n}$. For particular values of the variables lying on the topological locus $p_{n}=\frac{1-A^{n}}{1-q^{n}}$ Schur polynomials are given by very simple factorized formulas:

$$
s_{Y}\left(\frac{1-A^{n}}{1-t^{n}}\right)=\prod_{(i, j) \in Y} t^{i-1} \frac{1-A t^{j-i}}{1-t^{Y_{i}-j+Y_{j}^{\mathrm{T}}-i+1}}
$$

These expressions can be related to "quantum dimensions", or generating functions of the values of the Casimir operators on the corresponding representations.

Macdonald polynomials $M_{Y}^{(q, t)}\left(p_{n}\right)$ provide a natural generalization of Schur polynomials, depending on two parameters $q$ and $t$. Macdonald polynomials do not have immediate group theory interpretation, but nevertheless have many properties similar to Schur polynomials, to which they reduce for $t=q$. In particular, they still factorize on the topological locus $p_{n}=\frac{1-A^{n}}{1-t^{n}}$ :

$$
M_{Y}^{(q, t)}\left(\frac{1-A^{n}}{1-t^{n}}\right)=\prod_{(i, j) \in Y} t^{i-1} \frac{1-A q^{j-1} t^{1-i}}{1-q^{Y_{i}-j} t^{Y_{j}^{\mathrm{T}}-i+1}}
$$


Notice that the parameters of the topological locus for Macdonald polynomials are tied with the deformation parameters, so that for given $t$ the locus is one-dimensional. We will see similar effect in the following sections, where generalized Macdonald polynomials will depend on an additional parameter which will enter the definition of the topological locus.

\subsection{Generalized Macdonald polynomials factorization on the general topolog- ical locus}

In this section we give general factorization formulas for generalized Macdonald polynomials. Concretely, we have found a generalization of the factorization formula for generalized Macdonald polynomials conjectured in [61] to a wider topological locus. The identity reads: ${ }^{6}$

$$
\begin{aligned}
& M_{Y_{1} Y_{2}}^{(q, t)}\left(Q \mid \frac{1-A^{n}}{1-t^{n}}, \frac{\left(\frac{t}{q}\right)^{n}-1}{1-t^{n}}\right) \\
& =(-1)^{\left|Y_{2}\right|} t^{\frac{\left\|Y_{1}^{\mathrm{T}}\left|\|^{2}-\right| Y_{1} \mid\right.}{2}} q^{\frac{\| Y_{2}||^{2}-\left|Y_{2}\right|}{2}} \frac{G_{Y_{1} \varnothing}^{(q, t)}(A) G_{Y_{2} \varnothing}^{(q, t)}\left(A Q^{-1}\right) G_{\varnothing Y_{1}}^{(q, t)}\left(Q^{-1}\right) G_{\varnothing Y_{2}}^{(q, t)}(1)}{C_{Y_{1}}^{\prime}(q, t) C_{Y_{2}}^{\prime}(q, t) G_{Y_{2} Y_{1}}^{(q, t)}\left(Q^{-1}\right)}
\end{aligned}
$$

where

$$
\begin{aligned}
& G_{A B}^{(q, t)}(x)=\prod_{(i, j) \in A}\left(1-q^{x} q^{A_{i}-j} t^{B_{j}^{\mathrm{T}}-i+1}\right) \prod_{(i, j) \in B}\left(1-q^{x} q^{-B_{i}+j-1} t^{-A_{j}^{\mathrm{T}}+i}\right) \\
& C_{Y}^{\prime}(q, t)=\prod_{(i, j) \in Y}\left(1-q^{\operatorname{Arm}_{Y}(i, j)} t^{\operatorname{Leg}_{Y}(i, j)+1}\right), \quad|Y|=\sum_{i=1}^{l(Y)} Y_{i}, \quad\|Y\|^{2}=\sum_{i=1}^{l(Y)} Y_{i}^{2}
\end{aligned}
$$

The original formula which appeared in [61] is given by

$$
\begin{aligned}
& M_{Y_{1} Y_{2}}^{(q, t)}\left(Q \mid-\frac{1}{1-t^{n}}, 0\right) \\
& =(-1)^{\left|Y_{1}\right|} q^{\frac{|| Y_{1} \|^{2}-\left|Y_{1}\right|}{2}} Q^{-\left|Y_{2}\right|} q^{|| Y_{2} \|^{2}-\left|Y_{2}\right|} t^{-\frac{\left\|Y_{2}^{\mathrm{T}}\right\|^{2}-\left|Y_{2}\right|}{2}} \frac{G_{\varnothing Y_{1}}^{(q, t)}\left(Q^{-1}\right) G_{\varnothing Y_{2}}^{(q, t)}(1)}{C_{Y_{1}}^{\prime}(q, t) C_{Y_{2}}^{\prime}(q, t) G_{Y_{2} Y_{1}}^{(q, t)}\left(Q^{-1}\right)}
\end{aligned}
$$

It is obtained from eq. (2.4) in the limit $A \rightarrow \infty$ (one should divide both sides by $A^{\left|Y_{1}\right|+\left|Y_{2}\right|}$ to get a finite answer).

For completeness let us also give the factorization formula where the second argument of the generalized Macdonald polynomial is nontrivial:

$$
M_{Y_{1} Y_{2}}^{(q, t)}\left(Q \mid 0,-\frac{1-\left(\frac{t}{q} B\right)^{n}}{1-t^{n}}\right)=\delta_{Y_{1} \varnothing}(-1)^{\left|Y_{2}\right|} q^{\frac{\left\|Y_{2}\right\|^{2}-\left|Y_{2}\right|}{2}} \frac{G_{\varnothing Y_{2}}^{(q, t)}(B)}{C_{Y_{2}}^{\prime}(q, t)}
$$

\footnotetext{
${ }^{6}$ Similar identity for $A=Q$ has already appeared in [59] (see eq. (24) there). There was a minor typo in [59] eq. (24): Kronecker symbol $\delta_{Y_{2} \varnothing}$ was missing in the r.h.s.
} 
Notice the asymmetry between the two arguments of the generalized Macdonald polynomials: while the first factorization formula (2.4) has nontrivial dependence on both Young diagrams, the second one (2.8) actually reduces to the formula (2.3) for the ordinary Macdonald polynomials. This peculiar feature can be traced back to the nontrivial choice of coproduct in the DIM algebra [64, 65].

From the form of eqs. (2.4) and (2.8) one could have suspected that there is a general two-parametric factorization formula involving both $A$ and $B$. However, it turns out that this is not the case, as we explain below.

\subsection{Factorization identities from matrix model averages}

Before presenting more generalizations of the factorization formulas for generalized polynomials let us give here a short and simple proof for the factorization identities obtained so far. To this end we will employ the integral factorization identities discovered in [59, 60].

These identities give explicit factorized answers for $q$-Selberg averages of generalized Macdonald polynomials. The Selberg average of a symmetric function $f\left(x_{i}\right)$ is given by the following matrix integral:

$$
\left\langle f\left(x_{i}\right)\right\rangle_{u, v, N, q, t} \stackrel{\text { def }}{=} \frac{\int_{0}^{1} d_{q}^{N} x \mu\left(u, v, N, q, t \mid x_{i}\right) f\left(x_{i}\right)}{\int_{0}^{1} d_{q}^{N} x \mu\left(u, v, N, q, t \mid x_{i}\right)}
$$

where the integration measure is

$$
\mu\left(u, v, N, q, t \mid x_{i}\right)=\prod_{i \neq j} \prod_{k \geq 0} \frac{\left(\frac{x_{i}}{x_{j}} ; q\right)_{\infty}}{\left(t \frac{x_{i}}{x_{j}} ; q\right)_{\infty}} \prod_{i=1}^{N}\left(x_{i}^{u} \prod_{k \geq 0} \frac{\left(x_{i} ; q\right)_{\infty}}{\left(q^{v} x_{i} ; q\right)_{\infty}}\right)
$$

and the Jackson $q$-integral is defined as

$$
\int_{0}^{a} d_{q} x g(x)=(1-q) a \sum_{n \geq 0} q^{n} g\left(q^{n} a\right)
$$

One example of the factorized identity for the average considered in [60] is

$$
\begin{aligned}
& \left\langle M_{A B}^{(q, t)}\left(q^{-u-1} t \mid p_{-n}+\frac{(t / q)^{n}-q^{n v}}{1-t^{n}},-p_{-n}-\left(\frac{t}{q}\right)^{n} \frac{1-(t / q)^{n}}{1-t^{n}}\right)\right\rangle_{u, v, N, q, t}= \\
& =(-1)^{|A|} q^{-2|B|+u|A|} t^{|B|-|A|} t^{\sum_{(i, j) \in B} i+2 \sum_{(i, j) \in A} i} q^{-\sum_{(i, j) \in A}{ }^{j}} \times \\
& \times \frac{G_{A \varnothing}\left(t^{-N} q^{-u}\right) G_{A \varnothing}\left(t^{N-1} q^{v+1}\right) G_{B \varnothing}\left(t^{-N-1} q\right) G_{B \varnothing}\left(t^{N-2} q^{u+v+2}\right)}{C_{A}^{\prime}(q, t) C_{B}^{\prime}(q, t) G_{B A}^{(q, t)}\left(q^{u+1} t^{-1}\right)} .
\end{aligned}
$$

Notice that the parameters of the measure also enter the arguments of the generalized polynomials under the average sign. Let us make a peculiar specialization of eq. (2.12) and take $N=0$. What does it mean to have zero number of integrations? There is of course no general answer, but for Selberg averages the definition we consider seems very natural and can be obtained from analytic continuation in $N$. The generalized polynomial under 
the average is written in terms of power sums $p_{n}=\sum_{i=1}^{N} x_{i}^{n}$ of integration variables. For $N=0$ power sums contain zero terms and therefore should vanish. It is also evident that for $N=0$ there are no integrations neither in the numerator, nor in the denominator in the definition of the average and the integration measure is absent. Thus the l.h.s. of eq. (2.12) reduces to the generalized Macdonald polynomial evaluated at the point $p_{n}=0$, while the r.h.s. gives the correct factorized answer, coinciding with eq. (2.4).

Notice that the topological locus parametrized by $u$ and $v$ in eq. (2.12) and by $Q$ and $A$ in eq. (2.4) is two-dimensional. This will always be the case in our considerations since the original integral depends on three parameters, $u, v$, and $N$ and we have to put $N$ to zero.

More identities can be obtained by using the symmetry of the Selberg measure $\mu\left(u, v, N, q, t \mid x_{i}\right)$ under the change of parameters:

$$
\left(\begin{array}{c}
u \\
v \\
N
\end{array}\right) \rightarrow\left(\begin{array}{c}
\widetilde{u} \\
\widetilde{v} \\
\widetilde{N}
\end{array}\right)=\left(\begin{array}{c}
u \\
-v-2+2 \beta \\
N+\frac{v+1-\beta}{\beta}
\end{array}\right)
$$

Since $\mu\left(u, v, N, q, t \mid x_{i}\right)=\mu\left(\widetilde{u}, \widetilde{v}, \widetilde{N}, q, t \mid x_{i}\right)$, the average of any function $f(x)$ remains the same,

$$
\langle f(x)\rangle_{u, v, N, q, t}=\langle f(x)\rangle_{\widetilde{u}, \widetilde{v}, \widetilde{N}, q, t} .
$$

Of course, if the function $f$ itself depends on the parameters $u, v$ or $N$ one has to replace them with $\widetilde{u}, \widetilde{v}$ or $\widetilde{N}$ respectively to get the same average. Making the change of variables (2.13) in eq. (2.12) and setting $\widetilde{N}=0$ we get the identity (2.8).

Summarizing, the factorization identities for generalized Macdonald polynomials (2.4), (2.8) follow from the integral identity (2.12) in the limit $N=0$. In the next section we will give more factorization identities involving skew generalized Macdonald polynomials. They are proven using a similar argument.

\subsection{New formulas for skew generalized Macdonald polynomials}

We can also take the specialization $N=0$ in more general integral factorization formulas from [60] (see eqs. (93), (94) there). The identities we obtain in this way involve two skew generalized Macdonald polynomials. Skew generalized Macdonald polynomials are defined similarly to the usual skew Macdonald polynomials:

$$
M_{Y_{1} Y_{2} / Z_{1} Z_{2}}^{(q, t)}\left(Q \mid p_{n}, \bar{p}_{n}\right)=M_{Z_{1}}^{(q, t)}\left(n \frac{1-q^{n}}{1-t^{n}} \frac{\partial}{\partial p_{n}}\right) M_{Z_{2}}^{(q, t)}\left(n \frac{1-q^{n}}{1-t^{n}} \frac{\partial}{\partial \bar{p}_{n}}\right) M_{Y_{1} Y_{2}}^{(q, t)}\left(Q \mid p_{n}, \bar{p}_{n}\right)
$$


where $M_{Z}^{(q, t)}$ are ordinary Macdonald polynomials. Without giving too much technical details let us write down the final results:

$$
\begin{aligned}
& \sum_{Z_{1}, Z_{2}} \frac{\left(\frac{t}{q}\right)^{\left|Z_{1}\right|+\left|Z_{2}\right|}}{\|\left. M_{Z_{1}}||^{2}|| M_{Z_{2}}\right|^{2}} M_{Y_{1} Y_{2} / Z_{1} Z_{2}}^{*(q, t)}\left(Q \mid-\frac{1-\left(\frac{t}{q}\right)^{n}}{1-t^{n}},-\frac{1-B^{-n}}{1-t^{n}}\right) \times \\
& \times M_{W_{1} W_{2} / Z_{1} Z_{2}}^{(q, t)}\left(\frac{t}{q} B Q \mid 0, \frac{1-\left(\frac{t}{q} B\right)^{n}}{1-t^{-n}}\right)= \\
& =\left(\frac{t}{q B}\right)^{\left|Y_{1}\right|} t^{\frac{|| Y_{1}^{\mathrm{T}}\left|\|^{2}-\right| Y_{1} \mid}{2}}\left(-\frac{t Q}{q B}\right)^{\left|Y_{2}\right|} q^{-\frac{\| Y_{2}||^{2}-\left|Y_{2}\right|}{2}} t^{|| Y_{2}^{\mathrm{T}} \|^{2}-\left|Y_{2}\right|}(-t)^{\left|W_{1}\right|} q^{\frac{\| W_{1}||^{2}-\left|W_{1}\right|}{2}} \times \\
& \times\left(\frac{q}{B Q}\right)^{\left|W_{2}\right|} q^{|| W_{2}||^{2}-\left|W_{2}\right|} t^{-\frac{\left\|W_{2}^{\mathrm{T}}\right\|^{2}-\left|W_{2}\right|}{2}}\left(C_{Y_{1}}^{\prime}(q, t) C_{Y_{2}}^{\prime}(q, t) C_{W_{1}}^{\prime}(q, t) C_{W_{2}}^{\prime}(q, t)\right)^{-1} \times \\
& \times \frac{z_{\text {bifund }}^{\vec{Y}, \vec{W}}\left(Q^{\frac{1}{2}},\left(\frac{t}{q} B Q\right)^{\frac{1}{2}},\left(\frac{q}{t} B\right)^{-\frac{1}{2}}\right)}{G_{Y_{1} Y_{2}}^{(q, t)}(Q) G_{W_{2} W_{1}}^{(q, t)}\left(\left(\frac{t}{q} B Q\right)^{-1}\right)}
\end{aligned}
$$

where the conjugate generalized polynomial is defined as

$$
M_{Y_{1} Y_{2}}^{*(q, t)}\left(Q \mid p_{n}, \bar{p}_{n}\right)=M_{Y_{2} Y_{1}}^{(q, t)}\left(Q^{-1} \mid \bar{p}_{n}, p_{n}-\left(1-\left(\frac{t}{q}\right)^{n}\right) \bar{p}_{n}\right),
$$

and the norm of Macdonald polynomial is given by an explicit expression

$$
\left\|M_{Y}\right\|^{2}=\frac{C_{Y}^{\prime}(q, t)}{C_{Y}(q, t)} \quad C_{Y}(q, t)=\prod_{(i, j) \in Y}\left(1-q^{\operatorname{Arm}_{Y}(i, j)+1} t^{\operatorname{Leg}_{Y}(i, j)}\right)
$$

The bifundamental Nekrasov function is given by

$$
z_{\text {bifund }}^{\vec{Y}, \vec{W}}(Q, P, M)=G_{Y_{1} W_{1}}^{(q, t)}\left(\frac{Q}{M P}\right) G_{Y_{1} W_{2}}^{(q, t)}\left(\frac{Q P}{M}\right) G_{Y_{2} W_{1}}^{(q, t)}\left(\frac{1}{M Q P}\right) G_{Y_{2} W_{2}}^{(q, t)}\left(\frac{1}{M Q P}\right)
$$

There is one more identity similar to eq. (2.16):

$$
\begin{aligned}
& \sum_{Z_{1}, Z_{2}} \frac{\left(\frac{t}{q}\right)^{\left|Z_{1}\right|+\left|Z_{2}\right|}}{\|\left.\left. M_{Z_{1}}\right|^{2}|| M_{Z_{2}}\right|^{2}} M_{Y_{1} Y_{2} / Z_{1} Z_{2}}^{*(q, t)}\left(Q \mid-\frac{\left(\frac{t}{q}\right)^{n}-A^{-n}}{1-t^{-n}}, 0\right) \times \\
& \times M_{W_{1} W_{2} / Z_{1} Z_{2}}^{(q, t)}\left(\frac{t}{q} A Q \mid \frac{1-A^{n}}{1-t^{n}},-\frac{1-\left(\frac{t}{q}\right)^{n}}{1-t^{n}}\right)= \\
& =\left(\frac{t^{2}}{q}\right)^{\left|Y_{1}\right|} t^{\frac{|| Y_{1}^{\mathrm{T}}\left|\|^{2}-\right| Y_{1} \mid}{2}}\left(-\frac{Q t^{2}}{q}\right)^{\left|Y_{2}\right|} q^{-\frac{|| Y_{2}||^{2}-\left|Y_{2}\right|}{2}} t^{\left\|Y_{2}^{\mathrm{T}}\left|\|^{2}-\right| Y_{2} \mid\right.}(-A)^{\left|W_{1}\right|} q^{\frac{|| W_{1} \|^{2}-\left|W_{1}\right|}{2}} \times \\
& \times\left(\frac{q}{t Q}\right)^{\left|W_{2}\right|} q^{|| W_{2}||^{2}-\left|W_{2}\right|} t^{-\frac{\left\|W_{2}^{\mathrm{T}}\right\|^{2}-\left|W_{2}\right|}{2}}\left(C_{Y_{1}}^{\prime}(q, t) C_{Y_{2}}^{\prime}(q, t) C_{W_{1}}^{\prime}(q, t) C_{W_{2}}^{\prime}(q, t)\right)^{-1} \times \\
& \times \frac{z_{\text {bifund }}^{\vec{Y}, \vec{W}}\left(Q^{\frac{1}{2}},\left(\frac{t}{q} A Q\right)^{\frac{1}{2}},\left(\frac{t}{q} A\right)^{\frac{1}{2}}\right)}{G_{Y_{1} Y_{2}}^{(q, t)}(Q) G_{W_{2} W_{1}}^{(q, t)}\left(\frac{q}{t A Q}\right)}
\end{aligned}
$$


Identities (2.16), (2.20) are more general than eqs. (2.4), (2.8) and reduce to them in special cases. For $Y_{1,2}=\varnothing$ eq. (2.16) reduces to eq. (2.8) and for $W_{1,2}=\varnothing$ it reduces to eq. (2.4). In eq. (2.20) the situation is reversed, i.e. for $Y_{1,2}=\varnothing$ it reduces to eq. (2.4) and for $W_{1,2}=\varnothing$ it reduces to eq. (2.8).

\subsection{Gluing, traces and factorization of instanton sums}

The new identity (2.16) allows one to glue several factorized expressions together and then use Cauchy completeness to obtain a factorized answer for the full sum of factorized terms. As a simplest example we can take the trace over Young diagrams $\vec{Y}=\vec{W}$ in the identity (2.16). In the language of gauge theory this corresponds to making a circular quiver representing a $\mathrm{U}(2)$ adjoint theory, while for topological strings this gives the partial compactification of the base of the toric fibration. In each case, to get a meaningful result we have to set spectral parameters of the generalized Macdonald polynomials equal to each other. For eq. (2.16) this means taking $B=\frac{q}{t}$. Thus, we set $B=\frac{q}{t}, Y_{1}=W_{1}$ and $Y_{2}=W_{2}$ in eq. (2.16) and take the sum over Young diagrams $Y_{1,2}$ with weight $\frac{\Lambda^{\left|Y_{1}\right|+\left|Y_{2}\right|}}{\left\|M_{Y_{1}}\right\|^{2} \|\left. M_{Y_{2}}\right|^{2}}$. The r.h.s. of eq. (2.16) then takes the form of Nekrasov instanton partitions function for a particular value of the adjoint hypermultiplet mass:

$$
\begin{aligned}
& \sum_{Y_{1}, Y_{2}} \frac{\Lambda^{\left|Y_{1}\right|+\left|Y_{2}\right|}}{\left\|M_{Y_{1}}||^{2}|| M_{Y_{2}}\right\|^{2}} \sum_{Z_{1}, Z_{2}} \frac{\left(\frac{t}{q}\right)^{\left|Z_{1}\right|+\left|Z_{2}\right|}}{|| M_{Z_{1}} \|\left.^{2}|| M_{Z_{2}}\right|^{2}} M_{Y_{1} Y_{2} / Z_{1} Z_{2}}^{*(q, t)}\left(Q \mid-\frac{1-\left(\frac{t}{q}\right)^{n}}{1-t^{n}},-\frac{1-\left(\frac{t}{q}\right)^{n}}{1-t^{n}}\right) \times \\
& \times M_{Y_{1} Y_{2} / Z_{1} Z_{2}}^{(q, t)}(Q \mid 0,0)=\sum_{\vec{Y}}\left(\frac{t^{3}}{q^{3}} \Lambda\right)^{|\vec{Y}|} \frac{z_{\text {bifund }}^{\vec{Y}, \vec{Y}}\left(Q^{\frac{1}{2}}, Q^{\frac{1}{2}}, \frac{t}{q}\right)}{z_{\text {vect }}^{\vec{Y}}\left(Q^{\frac{1}{2}}\right)}
\end{aligned}
$$

Now we notice that the l.h.s. of eq. (2.21) does not depend on the choice of basis in the space of symmetric polynomials, since it is a trace over this space. This immediately implies that the l.h.s. is in fact independent of $Q$. Choosing the basis of ordinary Macdonald polynomials we find that the sum factorizes into a product of two identical sums:

$$
\begin{aligned}
& \sum_{Y_{1}, Y_{2}} \frac{\Lambda^{\left|Y_{1}\right|+\left|Y_{2}\right|}}{\left\|M_{Y_{1}}\right\|^{2}\left\|M_{Y_{2}}\right\|^{2}} \sum_{Z_{1}, Z_{2}} \frac{\left(\frac{t}{q}\right)^{\left|Z_{1}\right|+\left|Z_{2}\right|}}{\left\|M_{Z_{1}}\right\|^{2}\left\|M_{Z_{2}}\right\|^{2}} M_{Y_{1} Y_{2} / Z_{1} Z_{2}}^{*(q, t)}\left(Q \mid-\frac{1-\left(\frac{t}{q}\right)^{n}}{1-t^{n}},-\frac{1-\left(\frac{t}{q}\right)^{n}}{1-t^{n}}\right) \times \\
& \times M_{Y_{1} Y_{2} / Z_{1} Z_{2}}^{(q, t)}(Q \mid 0,0)=\left[\sum_{Y} \frac{\Lambda^{|Y|}}{\left\|M_{Y}\right\|^{2}} \sum_{Z} \frac{\left(\frac{t}{q}\right)^{|Z|}}{\left\|M_{Z}\right\|^{2}} M_{Y / Z}^{(q, t)}\left(-\frac{1-\left(\frac{t}{q}\right)^{n}}{1-t^{n}}\right) M_{Y / Z}^{(q, t)}(0)\right]^{2}
\end{aligned}
$$

One can immediately notice that

$$
M_{Y / Z}^{(q, t)}(0)=\delta_{Y Z}\left\|M_{Y}\right\|^{2}, \quad \text { and } \quad M_{Y / Y}^{(q, t)}\left(p_{n}\right)=\left\|M_{Y}\right\|^{2}
$$

so that the double sum in the r.h.s. turns into a single one:

$$
\sum_{Y} \frac{\Lambda^{|Y|}}{\left\|M_{Y}\right\|^{2}} \sum_{Z} \frac{\left(\frac{t}{q}\right)^{|Z|}}{\left\|M_{Z}\right\|^{2}} M_{Y / Z}^{(q, t)}\left(-\frac{1-\left(\frac{t}{q}\right)^{n}}{1-t^{n}}\right) M_{Y / Z}^{(q, t)}(0)=\sum_{Y}\left(\frac{t}{q} \Lambda\right)^{|Y|}=\prod_{k \geq 1} \frac{1}{1-\left(\frac{t}{q} \Lambda\right)^{k}}
$$


Eventually, we get the factorized answer for Nekrasov instanton partition function:

$$
\sum_{\vec{Y}}\left(\frac{t^{3}}{q^{3}} \Lambda\right)^{|\vec{Y}|} \frac{z_{\text {bifund }}^{\vec{Y}, \vec{Y}}\left(Q^{\frac{1}{2}}, Q^{\frac{1}{2}}, \frac{t}{q}\right)}{z_{\text {vect }}^{\vec{Y}}\left(Q^{\frac{1}{2}}\right)}=\prod_{k \geq 1} \frac{1}{\left(1-\left(\frac{t}{q} \Lambda\right)^{k}\right)^{2}}
$$

This is in fact nothing but the partition function of the corresponding $2 d$ CFT, which contains two bosonic field, hence power two in the r.h.s. The example we have described is of course a trivial one, since each term in the l.h.s. simplifies due to the identity

$$
z_{\text {bifund }}^{\vec{Y}, \vec{Y}}\left(Q^{\frac{1}{2}}, Q^{\frac{1}{2}}, \frac{t}{q}\right)=\left(\frac{q}{t}\right)^{2|\vec{Y}|} z_{\text {vect }}^{\vec{Y}}\left(Q^{\frac{1}{2}}\right)
$$

However, gluing two or more bifundamental contributions from eqs. (2.16), (2.20) together one gets nontrivial factorization identities for linear quiver gauge theories. Also one can take the trace to obtain circular quivers with several nodes.

\section{Toric brane on the intermediate leg and surface operators}

In this section we will demonstrate that factorization identities we have obtained can be thought of as the amplitudes of refined topological strings in the presence of a stack of toric branes. We will also comment on their relation with surface operators in gauge theory and degenerate vertex operators in $2 d$ CFT.

\subsection{Refined topological amplitudes with branes}

Selberg averages such as eq. (2.12), which we have used in our proof of factorization in section 2.3, can be identified with refined topological string amplitudes on toric CY depicted in figure 2. Kähler parameters $Q_{1,2}$ and $Q_{F}$ of the $\mathrm{CY}$ are related to the matrix integral parameters $u, v, N$ as follows:

$$
Q_{1}=t^{\frac{1}{2}-N} q^{\frac{1}{2}-v}, \quad Q_{2}=t^{\frac{1}{2}-N} q^{-\frac{1}{2}}, \quad Q_{F}=q^{u+v+\frac{3}{2}} t^{N-\frac{3}{2}}
$$

In eq. (3.1) one can also use $\widetilde{u}, \widetilde{v}$ and $\widetilde{N}$ obtained by the change of variables (2.13) instead of $u, v$ and $N$ to get the second possible identification between the parameters.

Factorization happens for a special value of the parameters corresponding to $N=0-$ the topological locus. On this locus one of the resolved conifold pieces in the toric diagram of the CY degenerates, i.e. its Kähler parameters becomes $\sqrt{\frac{q}{t}}$ or $\sqrt{\frac{t}{q}}$ as shown in figure 3 .

For unrefined amplitudes degenerate resolution factorizes into a product of two pieces as shown in figure 4 . Each piece is given by an explicit factorized formula, which coincides with the factorized answer for the polynomial. However, in the unrefined case the answers for the amplitudes from figure $4 \mathrm{~b}$ ) are not very interesting since generalized Macdonald polynomials in this case reduce to products of Schur functions, and the factorization identities turn into the well-known formulas for quantum dimensions (2.2). They reproduce the known amplitudes in the presence of the stack of toric branes on the intermediate leg in the unrefined theory. 


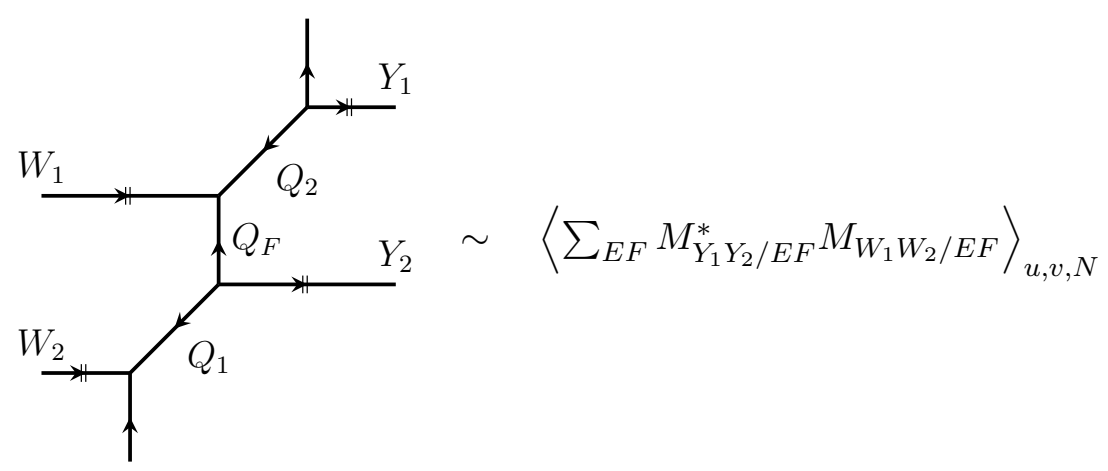

Figure 2. Refined topological string amplitude giving the $q$-Selberg average of two skew generalized Macdonald polynomials. The number of integrations $N$ and the parameters of the integral $u, v$ are expressed through the Kähler parameters $Q_{1,2}$ and $Q_{F}$ according to eq. (3.1).

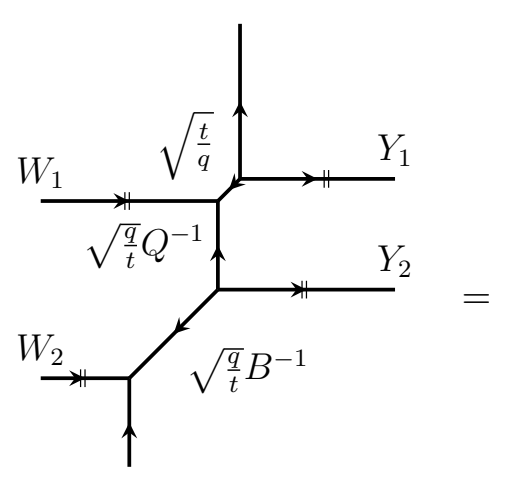

a)

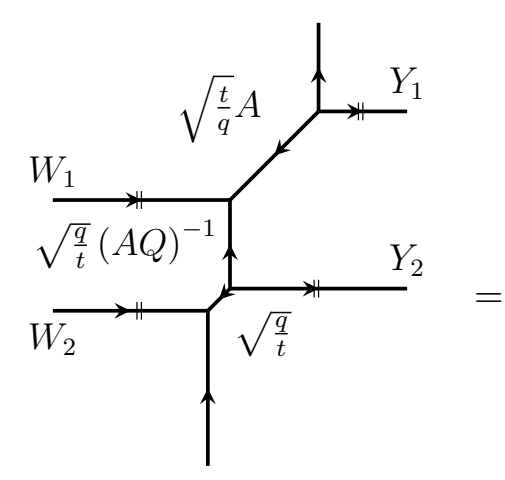

b)

Figure 3. a) Setting $Q_{1}=\sqrt{\frac{t}{q}}$ in the CY corresponds to setting $N=0$ in the Selberg average. For $Q_{2}=\sqrt{\frac{q}{t}} B^{-1}$ and $Q_{F}=\sqrt{\frac{q}{t}} Q^{-1}$ one arrives precisely at the factorization formula (2.16). The lower resolved conifold piece with Kähler parameter $\sqrt{\frac{q}{t}} B^{-1}$ can be transformed by geometric transition into geometry containing a stack of $M$ toric branes, where $t^{M}=\frac{q}{t} B^{-1}$. b) Setting $Q_{2}=\sqrt{\frac{q}{t}}$ in the $\mathrm{CY}$ corresponds to setting $\beta N=-v$ in the Selberg average. This average is related by the symmetry (2.13) to the integral with $N=0$ number of integrations shown in a). For $Q_{1}=\sqrt{\frac{t}{q}} A$ and $Q_{F}=\sqrt{\frac{q}{t}}(A Q)^{-1}$ one arrives at the factorization formula (2.20). This amplitudes corresponds to a stack of $M^{\prime}$ toric branes on the upper horizontal leg with $M^{\prime}$ given by $t^{M^{\prime}}=A$.

For refined amplitudes degenerate conifold geometry does not split into two parts. Moreover, there are two different degenerations of the resolved conifold with Kähler parameter either $\sqrt{\frac{q}{t}}$ or $\sqrt{\frac{t}{q}}$ as shown in figure $5 \mathrm{a}$ ). The difference between these two situations is evident from figure $5 \mathrm{~b}$ ) and c): when some of the legs are empty the amplitudes do factorize and give the same result as in the unrefined case.

The amplitudes from figure 3 are still given by the factorized expressions, though they cannot be separated into two noninteracting parts as shown in figure $4 \mathrm{~b}$ ). We argue that this is the natural definition of the stack of toric branes placed on the horizontal leg of the diagrams. For a single horizontal leg the corresponding geometry is shown in 
a)

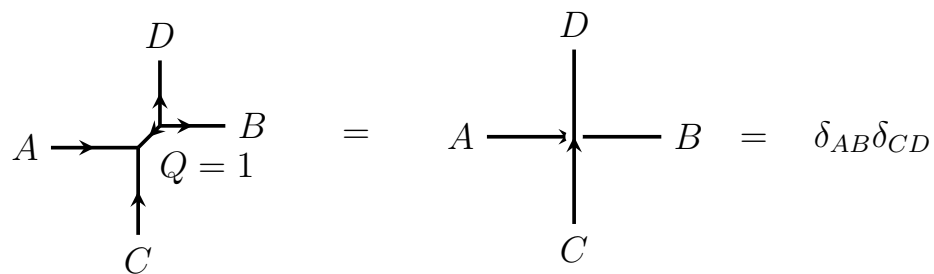

b)

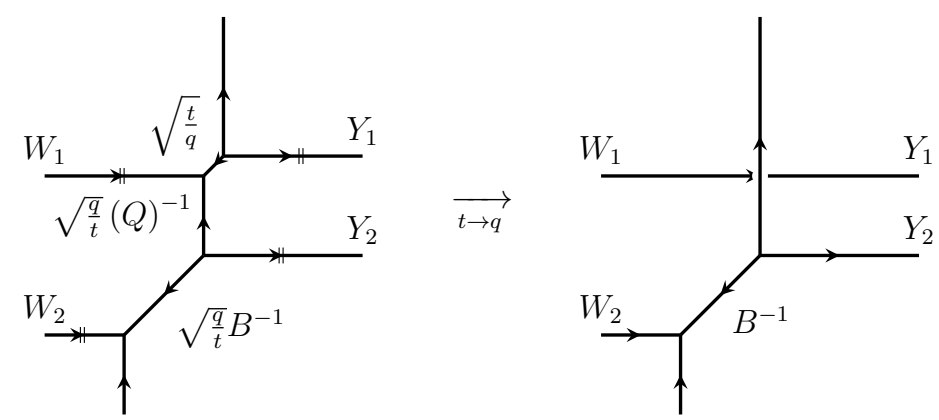

Figure 4. a) Unrefined amplitude on the resolved conifold in the degenerate limit $Q \rightarrow 1$ factorizes into a product of two separate non-interacting lines. Notice that there is no preferred direction in the unrefined case. b) Though the values of the polynomials on the topological locus are factorized into a product of monomials, they do not factorize into a product of independent terms corresponding to two horizontal lines. This happens only in the unrefined limit $t \rightarrow q$.

a)

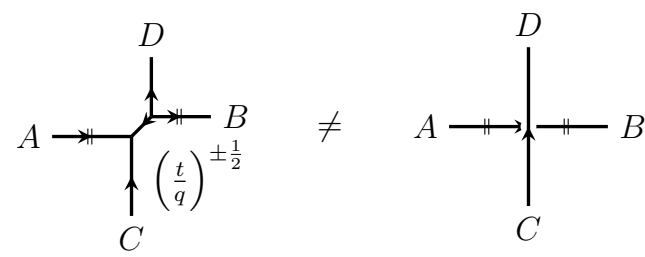

b)

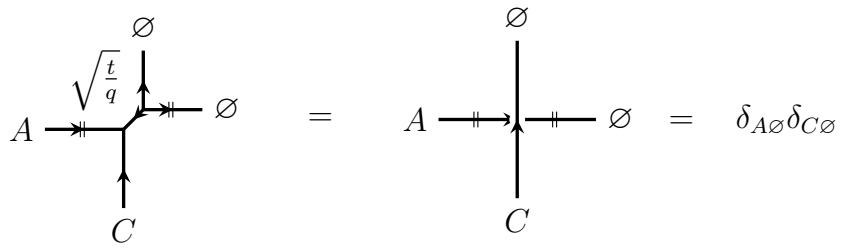

c)

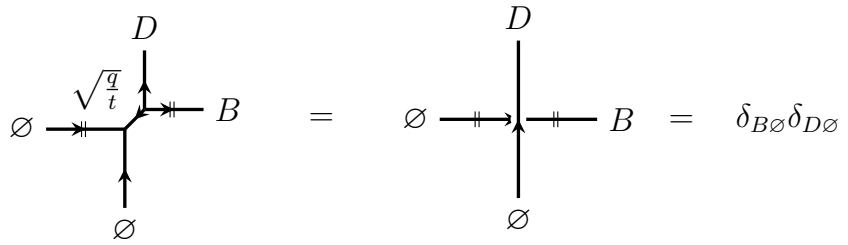

Figure 5. a) Amplitudes on degenerate resolved conifold do not trivialize. However, if some of the diagrams are empty, the amplitude reduces to the unresolved one. For two choices of Kähler parameter $\left(\frac{t}{q}\right)^{ \pm \frac{1}{2}}$ one gets different decoupling conditions, b) and c). 


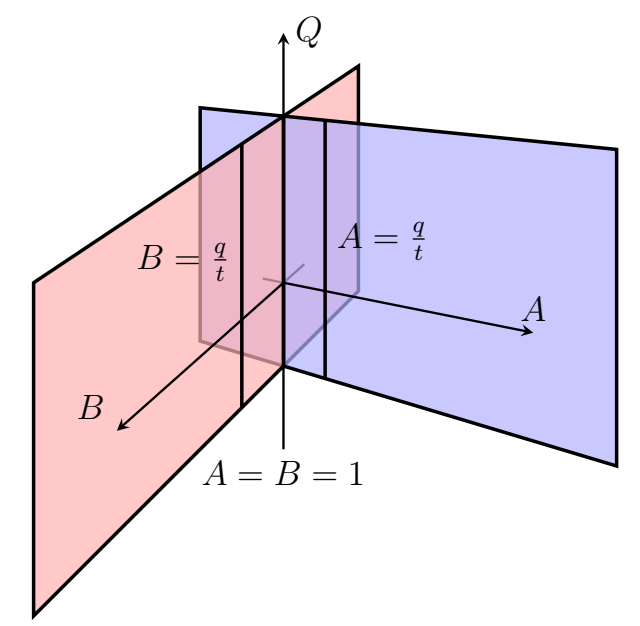

Figure 6. Schematic representation of the topological loci inside the Kähler moduli space of the strip geometry which can also be thought of as Coulomb moduli space of the gauge theory. The moduli space is parametrized by $Q, A$ and $B$, which are transformed into $Q_{1}, Q_{2}$ and $Q_{F}$ using the formulas $Q_{1}=\sqrt{\frac{t}{q}} A, Q_{2}=\sqrt{\frac{q}{t}} B^{-1}, Q_{F}=\sqrt{\frac{q}{t}}(Q A)^{-1}$. There are two distinct topological loci, $\{A=1\}$ and $\{B=1\}$ (shown in red and blue respectively), which intersect on the line $A=B=1$. There are also two special lines, $A=\frac{q}{t}$ and $B=\frac{q}{t}$, each on its own locus, where and $Q_{1}=Q_{2}=\left(\frac{q}{t}\right)^{ \pm 1}$. In the unrefined limit $t \rightarrow q$ the special lines coalesce with the intersection of the two loci.

eq. (1.5). However, if we have several horizontal legs, the vertical line will intersect several of them. Figure 3 describes precisely this situation: figure 3 a) models the stack of $M$ branes on the lower horizontal line, and figure $3 \mathrm{~b}$ ) represents the stack of $M^{\prime}$ branes on the upper horizontal line. The intersection of the vertical line with the second horizontal leg is degenerate and in the unrefined limit gives the trivial crossing from figure 4 . In the refined case the crossing trivializes when the corresponding diagram on the left or right of the crossing is empty as depicted in figure $5 \mathrm{~b}$ ), c).

Let us recapitulate our main point. A stack of refined toric branes sitting on a preferred leg of the diagram interacts with all other parallel legs. The resulting amplitude is given by the factorized value of generalized Macdonald polynomials evaluated on the topological locus.

\subsection{Degenerate fields and surface operators}

Topological loci can be given a natural gauge theory interpretation. The Kähler moduli space of the CY is identified with the Coulomb moduli space of the $5 d$ gauge theory. The topological locus corresponds to the root of the Higgs branch inside the Coulomb branch. In other words, degeneration of the resolved conifold pieces of the toric diagram allows one to deform the geometry instead. This deformation corresponds to going on the Higgs branch.

The identification can also be seen directly by identifying the parameters of the corresponding gauge theory with Kähler parameters of the CY. The geometry in figure 2 corresponds to a single bifundamental field of mass $m$ charged under two $\mathrm{SU}(2)$ gauge groups, 
$\mathrm{SU}(2)_{L}$ and $\mathrm{SU}(2)_{R}$. The parameters of the theory are the Coulomb moduli $Q_{L}=q^{a_{L}}$, $Q_{R}=q^{a_{R}}$ and the exponentiated mass $Q_{m}=q^{m}$. They are given by the following formulas:

$$
Q_{L}=\left(Q_{1} Q_{F}\right)^{\frac{1}{2}}, \quad Q_{R}=\left(Q_{F} Q_{2}^{-1}\right)^{\frac{1}{2}}, \quad Q_{m}=\sqrt{\frac{t}{q}}\left(Q_{1} Q_{2}^{-1}\right)^{\frac{1}{2}}
$$

One can immediately see that on the topological locus where we have either $Q_{L}=Q_{R} Q_{m}$ or $Q_{R}=Q_{L} Q_{m}$. This indeed corresponds to the origin of Higgs branches.

It is well-known that gauge theory at this point is equivalent to a theory on a defect associated with surface operator. Factorization formulas allow us to identify partition function of this theory with the values of the generalized Macdonald polynomials on the topological locus.

One final interpretation of the factorization formulas is given by the thedegenerate vertex operators in $q$-deformed $2 d$ CFT. According to the AGT relations, gauge theory we have just described corresponds to vertex operator in the Liouville theory. The Selberg integrals used in section 2.3 are interpreted as integrals in the DF screening charges. Naturally, if $N=0$, the screening charges are absent and we return to pure bosonic vertex operator

$V_{\frac{Q_{1}}{Q_{2}}}$. Matrix elements of this operator in the generalized Macdonald basis are given by generalized Macdonald polynomials evaluated on the topolgical locus. Schematically this can be written as follows:

$$
\left\langle M_{Y_{1} Y_{2}}^{*}\left(Q_{R}\right)\left|V_{Q_{m}}\right| M_{W_{1} W_{2}}\left(Q_{R} Q_{m}\right)\right\rangle=\left.\sum_{Z_{1} Z_{2}} M_{Y_{1} Y_{2} / Z_{1} Z_{2}}^{*}\left(Q_{R}\right) M_{W_{1} W_{2} / Z_{1} Z_{2}}\left(Q_{R} Q_{m}\right)\right|_{\text {top locus }}
$$

As usual degenerate field obeys a difference equation. Thus, generalized Macdonald polynomials taken on the topological locus should also obey this equation. We hope to clarify this point in the future.

\section{Conclusions and further prospects}

In this paper we have presented new factorization identities for generalized Macdonald polynomials. We proved the identities using the technique of matrix models and related them to refined topological string amplitudes in the presence of a stack of toric branes. We have also identified the corresponding gauge theories and CFT vertex operators.

It would be interesting to understand better the meaning of the factorization identities directly in the DIM algebra. Also we would like to investigate the difference equations satisfied by the polynomials on the topological locus. Nekrasov-Shatashvili limit of our construction might help to understand better the surface operators corresponding to toric branes on the intermediate legs of the toric diagram.

\section{Acknowledgments}

The author thanks Y. Kononov and N. Sopenko for discussions. The work of the author was supported in part by INFN, by the ERC Starting Grant 637844-HBQFTNCER and by RFBR grants 17-01-00585, 15-31-20484-mol-a-ved, 15-51-52031_NSC, 15-51-50034_YaF, 16-51-53034_GFEN, 16-51-45029_Ind. 
Open Access. This article is distributed under the terms of the Creative Commons Attribution License (CC-BY 4.0), which permits any use, distribution and reproduction in any medium, provided the original author(s) and source are credited.

\section{References}

[1] A. Iqbal, All genus topological string amplitudes and five-brane webs as Feynman diagrams, hep-th/0207114 [INSPIRE].

[2] M. Aganagic, A. Klemm, M. Marino and C. Vafa, The Topological Vertex, Commun. Math. Phys. 254 (2005) 425 [hep-th/0305132] [INSPIRE].

[3] A. Okounkov, N. Reshetikhin and C. Vafa, Quantum Calabi-Yau and Classical Crystals, hep-th/0309208 [INSPIRE].

[4] T. Eguchi and H. Kanno, Topological strings and Nekrasov's formulas, JHEP 12 (2003) 006 [hep-th/0310235] [INSPIRE].

[5] A. Iqbal, N. Nekrasov, A. Okounkov and C. Vafa, Quantum foam and topological strings, JHEP 04 (2008) 011 [hep-th/0312022] [INSPIRE].

[6] R. Dijkgraaf, C. Vafa and E. Verlinde, M-theory and a topological string duality, hep-th/0602087 [INSPIRE].

[7] T.J. Hollowood, A. Iqbal and C. Vafa, Matrix models, geometric engineering and elliptic genera, JHEP 03 (2008) 069 [hep-th/0310272] [INSPIRE].

[8] H. Awata and H. Kanno, Instanton counting, Macdonald functions and the moduli space of D-branes, JHEP 05 (2005) 039 [hep-th/0502061] [INSPIRE].

[9] A. Iqbal, C. Kozcaz and C. Vafa, The Refined topological vertex, JHEP 10 (2009) 069 [hep-th/0701156] [INSPIRE].

[10] J. Ding and K. Iohara, Generalization of Drinfeld quantum affine algebras, Lett. Math. Phys. 41 (1997) 181 [q-alg/9608002].

[11] K. Miki, $A(q, \gamma)$ analog of the $W_{1+\infty}$ algebra, J. Math. Phys. 48 (2007) 123520.

[12] H. Nakajima and K. Yoshioka, Instanton counting on blowup. 1., Invent. Math. 162 (2005) 313 [math/0306198] [INSPIRE].

[13] H. Nakajima and K. Yoshioka, Lectures on instanton counting, math/0311058 [INSPIRE].

[14] H. Nakajima and K. Yoshioka, Instanton counting on blowup. II. K-theoretic partition function, math/0505553 [INSPIRE].

[15] A. Losev, N. Nekrasov and S.L. Shatashvili, Issues in topological gauge theory, Nucl. Phys. B 534 (1998) 549 [hep-th/9711108] [INSPIRE].

[16] A. Losev, N. Nekrasov and S.L. Shatashvili, Testing Seiberg-Witten Solution, hep-th/9801061 [INSPIRE].

[17] G.W. Moore, N. Nekrasov and S. Shatashvili, Integrating over Higgs branches, Commun. Math. Phys. 209 (2000) 97 [hep-th/9712241] [INSPIRE].

[18] G.W. Moore, N. Nekrasov and S. Shatashvili, D-particle bound states and generalized instantons, Commun. Math. Phys. 209 (2000) 77 [hep-th/9803265] [INSPIRE]. 
[19] N.A. Nekrasov, Seiberg-Witten prepotential from instanton counting, Adv. Theor. Math. Phys. 7 (2003) 831 [hep-th/0206161] [INSPIRE].

[20] R. Flume and R. Poghossian, An Algorithm for the microscopic evaluation of the coefficients of the Seiberg-Witten prepotential, Int. J. Mod. Phys. A 18 (2003) 2541 [hep-th/0208176] [INSPIRE].

[21] N. Nekrasov and A. Okounkov, Seiberg-Witten theory and random partitions, Prog. Math. 244 (2006) 525 [hep-th/0306238] [INSPIRE].

[22] A. Mironov and A. Morozov, The Power of Nekrasov Functions, Phys. Lett. B 680 (2009) 188 [arXiv:0908.2190] [INSPIRE].

[23] L.F. Alday, D. Gaiotto and Y. Tachikawa, Liouville Correlation Functions from Four-dimensional Gauge Theories, Lett. Math. Phys. 91 (2010) 167 [arXiv:0906.3219] [INSPIRE].

[24] N. Wyllard, $A_{N-1}$ conformal Toda field theory correlation functions from conformal $N=2$ $\mathrm{SU}(N)$ quiver gauge theories, JHEP 11 (2009) 002 [arXiv:0907.2189] [INSPIRE].

[25] A. Mironov and A. Morozov, On AGT relation in the case of $\mathrm{U}(3)$, Nucl. Phys. B $\mathbf{8 2 5}$ (2010) 1 [arXiv:0908.2569] [INSPIRE].

[26] H. Awata and Y. Yamada, Five-dimensional AGT Conjecture and the Deformed Virasoro Algebra, JHEP 01 (2010) 125 [arXiv:0910.4431] [INSPIRE].

[27] H. Awata and Y. Yamada, Five-dimensional AGT Relation and the Deformed beta-ensemble, Prog. Theor. Phys. 124 (2010) 227 [arXiv:1004.5122] [InSPIRE].

[28] M.-C. Tan, M-Theoretic Derivations of 4d-2d Dualities: From a Geometric Langlands Duality for Surfaces, to the AGT Correspondence, to Integrable Systems, JHEP 07 (2013) 171 [arXiv:1301.1977] [INSPIRE].

[29] M.-C. Tan, An M-Theoretic Derivation of a $5 d$ and $6 d$ AGT Correspondence and Relativistic and Elliptized Integrable Systems, JHEP 12 (2013) 031 [arXiv:1309.4775] [INSPIRE].

[30] M.-C. Tan, Higher AGT Correspondences, W-algebras and Higher Quantum Geometric Langlands Duality from M-theory, arXiv: 1607.08330 [INSPIRE].

[31] A. Iqbal, C. Kozcaz and S.-T. Yau, Elliptic Virasoro Conformal Blocks, arXiv:1511.00458 [INSPIRE].

[32] F. Nieri, An elliptic Virasoro symmetry in 6d, arXiv:1511.00574 [INSPIRE].

[33] A. Nedelin and M. Zabzine, q-Virasoro constraints in matrix models, JHEP 03 (2017) 098 [arXiv: 1511.03471] [INSPIRE].

[34] R. Yoshioka, The integral representation of solutions of $K Z$ equation and a modification by $\mathcal{K}$ operator insertion, arXiv: 1512.01084 [INSPIRE].

[35] A. Mironov, A. Morozov and Y. Zenkevich, On elementary proof of AGT relations from six dimensions, Phys. Lett. B 756 (2016) 208 [arXiv:1512.06701] [InSPIRE].

[36] A. Mironov, A. Morozov and Y. Zenkevich, Spectral duality in elliptic systems, six-dimensional gauge theories and topological strings, JHEP 05 (2016) 121 [arXiv: 1603.00304] [INSPIRE].

[37] E. Carlsson, N. Nekrasov and A. Okounkov, Five dimensional gauge theories and vertex operators, Moscow Math. J. 14 (2014) 39 [arXiv:1308.2465] [INSPIRE]. 
[38] H. Awata, B. Feigin and J. Shiraishi, Quantum Algebraic Approach to Refined Topological Vertex, JHEP 03 (2012) 041 [arXiv: 1112.6074] [INSPIRE].

[39] H. Awata, B. Feigin, A. Hoshino, M. Kanai, J. Shiraishi and S. Yanagida, Notes on Ding-Iohara algebra and AGT conjecture, arXiv:1106.4088 [INSPIRE].

[40] A. Mironov, A. Morozov and Y. Zenkevich, Ding-Iohara-Miki symmetry of network matrix models, Phys. Lett. B 762 (2016) 196 [arXiv:1603.05467] [InSPIRE].

[41] H. Awata et al., Explicit examples of DIM constraints for network matrix models, JHEP 07 (2016) 103 [arXiv : 1604.08366] [INSPIRE].

[42] J.-E. Bourgine, Y. Matsuo and H. Zhang, Holomorphic field realization of $S H^{c}$ and quantum geometry of quiver gauge theories, JHEP 04 (2016) 167 [arXiv:1512.02492] [INSPIRE].

[43] M. Aganagic and S. Shakirov, Refined Chern-Simons Theory and Topological String, arXiv: 1210.2733 [INSPIRE].

[44] L.F. Alday, D. Gaiotto, S. Gukov, Y. Tachikawa and H. Verlinde, Loop and surface operators in $N=2$ gauge theory and Liouville modular geometry, JHEP 01 (2010) 113 [arXiv:0909.0945] [INSPIRE].

[45] D. Gaiotto, Surface Operators in $N=2$ 4d Gauge Theories, JHEP 11 (2012) 090 [arXiv:0911.1316] [INSPIRE].

[46] H. Awata, H. Fuji, H. Kanno, M. Manabe and Y. Yamada, Localization with a Surface Operator, Irregular Conformal Blocks and Open Topological String, Adv. Theor. Math. Phys. 16 (2012) 725 [arXiv: 1008.0574] [inSPIRE].

[47] A. Marshakov, A. Mironov and A. Morozov, On AGT Relations with Surface Operator Insertion and Stationary Limit of Beta-Ensembles, J. Geom. Phys. 61 (2011) 1203 [arXiv: 1011.4491] [INSPIRE].

[48] M. Aganagic, M.C.N. Cheng, R. Dijkgraaf, D. Krefl and C. Vafa, Quantum Geometry of Refined Topological Strings, JHEP 11 (2012) 019 [arXiv:1105.0630] [INSPIRE].

[49] H.-Y. Chen and A. Sinkovics, On Integrable Structure and Geometric Transition in Supersymmetric Gauge Theories, JHEP 05 (2013) 158 [arXiv:1303.4237] [INSPIRE].

[50] H. Mori and Y. Sugimoto, Surface Operators from M-strings, Phys. Rev. D 95 (2017) 026001 [arXiv: 1608.02849] [INSPIRE].

[51] A. Iqbal and C. Vafa, BPS Degeneracies and Superconformal Index in Diverse Dimensions, Phys. Rev. D 90 (2014) 105031 [arXiv:1210.3605] [InSPIRE].

[52] J. Gomis and T. Okuda, D-branes as a Bubbling Calabi-Yau, JHEP 07 (2007) 005 [arXiv: 0704.3080] [INSPIRE].

[53] C. Kozcaz, S. Pasquetti and N. Wyllard, A \& B model approaches to surface operators and Toda theories, JHEP 08 (2010) 042 [arXiv: 1004.2025] [INSPIRE].

[54] T. Dimofte, S. Gukov and L. Hollands, Vortex Counting and Lagrangian 3-manifolds, Lett. Math. Phys. 98 (2011) 225 [arXiv:1006.0977] [INSPIRE].

[55] M. Taki, Surface Operator, Bubbling Calabi-Yau and AGT Relation, JHEP 07 (2011) 047 [arXiv: 1007.2524$]$ [INSPIRE].

[56] Y. Ohkubo, Existence and Orthogonality of Generalized Jack Polynomials and Its q-Deformation, J. Phys. Conf. Ser. 804 (2017) 012036 [arXiv: 1404.5401] [INSPIRE]. 
[57] M. Aganagic, N. Haouzi, C. Kozcaz and S. Shakirov, Gauge/Liouville Triality, arXiv: 1309.1687 [INSPIRE].

[58] M. Aganagic, N. Haouzi and S. Shakirov, $A_{n}$-Triality, arXiv: 1403.3657 [INSPIRE].

[59] Y. Zenkevich, Generalized Macdonald polynomials, spectral duality for conformal blocks and AGT correspondence in five dimensions, JHEP 05 (2015) 131 [arXiv:1412.8592] [INSPIRE].

[60] A. Morozov and Y. Zenkevich, Decomposing Nekrasov Decomposition, JHEP 02 (2016) 098 [arXiv: 1510.01896] [INSPIRE].

[61] Ya. Kononov and A. Morozov, On Factorization of Generalized Macdonald Polynomials, Eur. Phys. J. C 76 (2016) 424 [arXiv:1607.00615] [InSPIRE].

[62] B. Feigin, M. Jimbo, T. Miwa and E. Mukhin, Quantum toroidal $\mathfrak{g l}_{1}$ and Bethe ansatz, J. Phys. A 48 (2015) 244001 [arXiv: 1502.07194] [InSPIRE].

[63] B. Feigin, M. Jimbo, T. Miwa and E. Mukhin, Finite type modules and Bethe Ansatz for quantum toroidal gl(1), arXiv:1603.02765 [INSPIRE].

[64] H. Awata et al., Toric Calabi-Yau threefolds as quantum integrable systems. $\mathcal{R}$-matrix and $\mathcal{R} \mathcal{T} \mathcal{T}$ relations, JHEP 10 (2016) 047 [arXiv: 1608.05351] [INSPIRE].

[65] H. Awata et al., Anomaly in RTT relation for DIM algebra and network matrix models, Nucl. Phys. B 918 (2017) 358 [arXiv:1611.07304] [INSPIRE]. 